\title{
A Security System Based on Remote Iris Recognition
}

\author{
Sha Lu, Huaideng Wang, Jing He \\ School of Information Science and Engineering \\ Jinling College, Nanjing University \\ Nanjing, China, 210089
}

\author{
Jie Yuan* \\ School of Electronic Science and Engineering \\ Nanjing University \\ Nanjing, China, 210093 \\ *Correspondent Author: yuanjie@nju.edu.cn
}

\begin{abstract}
In this paper, we present a remote iris recognition system using two cooperative cameras, one of which with wideangle perspective and the other with long focal-length and high resolution; the two cameras are fixed together on a resolving stage called Pan and Tilt. When the wide-angle camera finds a face, it locates the eye and instructs the Pan and Tilt to turn correspondingly, so as to allow the high resolution camera to see the eye as well. The technique allows the high resolution camera capture an iris image from a remote distance then to carry out regular iris recognition procedure. Due to the fusion of face tracking and iris recognition, the system achieves recognition accuracy up to $95 \%$, at a working distance of over $100 \mathrm{~cm}$.
\end{abstract}

Keywords-remote iris recognition, cooperative cameras, face tracking

\section{INTRODUCTION}

With an increasingly significant emphasis on security in the information-based society, personal authentication is receiving more and more attention. Automated identification based on biometrics, considered to be the most unique, robust and stable, has developed rapidly. Iris recognition, one of the most important biometric authentications, is becoming an active topic in both research and practical fields, because it is very hard to fabricate compared with the other human traits such as handwriting, fingerprint, voice. In the practical field, iris recognition system can only work in a relative short distance, $40 \mathrm{~cm}$ or less based on our latest Internet search. These products all require the testers' cooperation and concentration. In laboratories, some successful experiments which can capture an iris image from beyond over $1 \mathrm{~cm}$ are based on extremely high qualified camera. This paper presents a design to implement iris cognition from a remote distance, around 1.5 meters. The system is assembled with two cameras: one is a wideangle camera called assistant camera, and the other is a high resolution camera which serves as the main camera. To make the system work well, a set of image processing algorithms are deliberately designed.

Compared with the traditional design, our system has superiorities as follow:

Innovatively designed a remote vision system assembled with two optical axis paralleled cameras, where the wide-angle camera helps detect human face and target eyes in a wide-angle perspective and the long focallength camera capture iris images. The user does not need to worry about their height, or stand right in front of the system for the equipment can work in a scope of 120 degree in horizontal direction and 60 degree in vertical direction.

Long iris image acquisition distance, up to $200 \mathrm{~cm}$. Accuracy up to $95 \%$ is achieved at 1 meter, while we lost some accuracy at 2 meters. Besides angle scale of user tracking is up to $355^{\circ}$.Users may stand casually or even move slowly.

Rich of features such as remote monitoring, network databases, voice notification, touch screen and SMS delivering, which form a practical utility. The system can be easily applied to Access Control and Information Dissemination Area in a company.

\section{SYSTEM OVERVIEW}

The system comprises of 5 parts: 1. multiprocessor platform; 2. image acquisition part; 3. user interface; 4. system monitor and control; 5. network SQL server. Each part works individually and part 2 5 are all connected with part 1 which is the core of the whole system. The system map is shown in Figure 1.

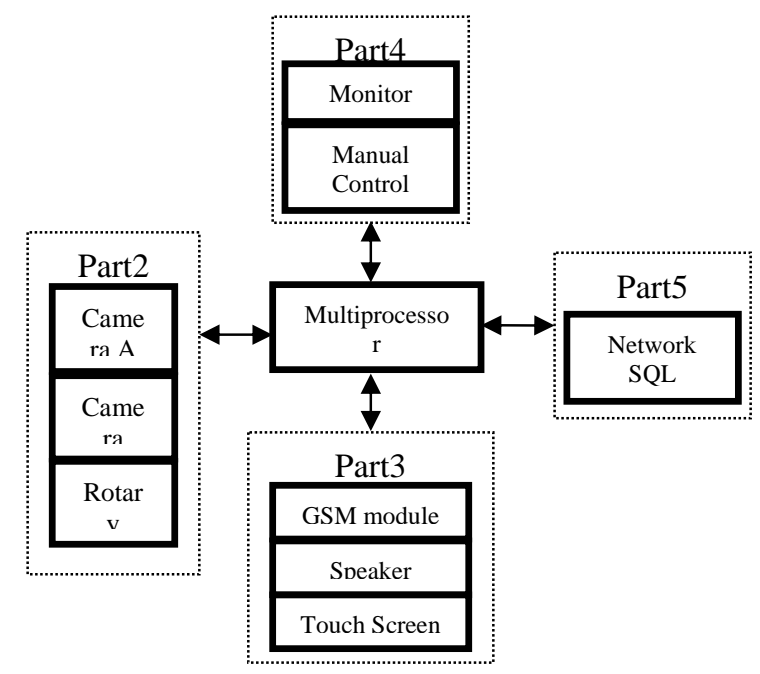

Figure 1. System Map:5 parts of the system and their relationship 
Part 1 takes most of the computing tasks including image processing, device control etc. An operating system is embedded in the platform. This platform also provides necessary standard interfaces for other parts. Part 2 comprises of 2 CCD digital cameras and a resolving stage called Pan and Tilt. One camera has wide optic-angle (as assistant camera below), the other camera has long focallength and high resolution (as high-resolution camera below).The Pan and Tilt rotates both vertically and horizontally, and can be controlled by part 1 with certain instructions. Both cameras are fixed on the Pan and Tilt like shown in Figure2. Part 3 includes touch screen, speaker and a GSM module to send short messages. Part 4 includes a monitor and some general inputs. Part 5 is connected with part 1 through network.

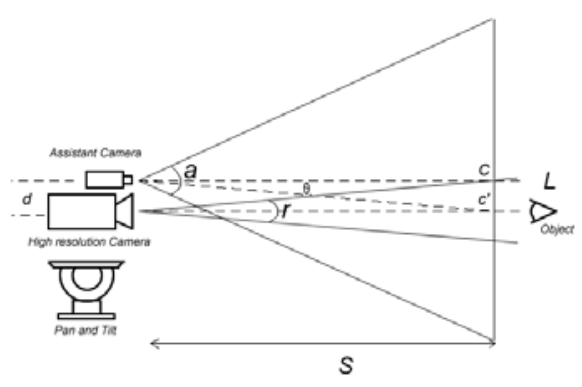

Figure 2. The image acquisition part

The functions of the system are listed as follows:

a) Real-time face tracking and eye positioning: Face detection and eye positioning can be processed in the rate of $30 \mathrm{fps}$. So the visitor's eye is tracked dynamically and can be locked in 5 seconds. The system functions well against illumination and under multi-people situation.

b) Iris image acquisition and recognition: the system detects a human face automatically and tracks it, after a certain process the visitor's iris will be recognized.

c) User interactive: This part takes responsibility of answering for visitor's request and give prompt. Also visitors can make a quick registration into the system.

d) Local administration: Administrators of the system can monitor the status of the system, including details of face tracking and iris recognition. Administrators can make necessary adjustment of parameters, such as optical adjustment, skin color threshold and available scales of rotary table.

e) Network identification database: Identification database is published by a network SQL server. All similar systems share 1 database on this server.

When a visitor wants to get into the security system, one could use the touch screen to send a signal to the system. After that the system starts its process automatically: 1 . the assistant camera captures an image and judges where the human face is. 2. Then human eyes is also positioned; 3. Based on the position of the human eyes, the system controls the Pan and Tilt in order to focus one human eye. 3. After a close loop control process, the high-resolution camera captures an image with a human eye, which takes more than $60 \%$ of whole image. In this image the eyeball can be accurately positioned; 4.The last step is to get the iris image from the eyeball image and recognize it. If this visitor has been registered, the touch screen displays information of the matching result and a welcome message is sent to the visitor's cell, otherwise this visitor will be rejected.

\section{IMPLEMENTATION OF ALGORITHM}

\section{A. Face Tracking}

Generally, this part follows 3 steps (i) Skin Region Detection, (ii) Face Candidate Localization (iii) Final Decision. In step (i) the source image is transformed from RGB color space into $\mathrm{YCbCr}$ color space, and the skin region can be segmented. Finally we get a binary image in this step. In step (ii) Median filter and morphological closing are applied to the binary image. All connected regions are located after a global scanning. In step (iii) any region without right geometry or area is eliminated, the largest region of the rest is the human face region. After that the rotary table moves to ensure human face region at the center of the whole image.

\section{B. Eye Positioning}

According to a face gray image, it is easily to Figureure out that eyes are in the least brightness and have the most details. In another word, eyes have a low brightness value and a high gradient value at the same. To joint analyses of gray images and gradient images, we applied a union evaluate function as follow:

$$
I(x, y)=\frac{G(x, y)}{S(x, y)}
$$

Then we divided the gradient value by a gray value at each pixel, thus the eye pixels were easily found for their values were much higher. Meanwhile, we also followed the geometry characteristic of faces to exclude eyebrows and nostrils, and consequently precisely targeted the eye. The transformed images are shown in Figure.3. Analysis on image I is shown in Figure4.

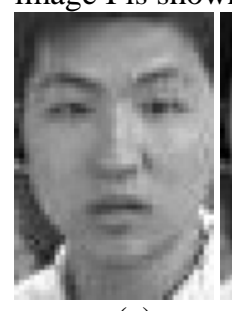

(a)

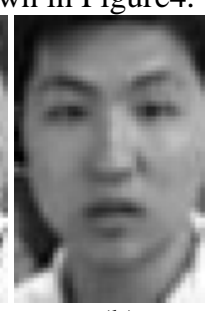

(b)

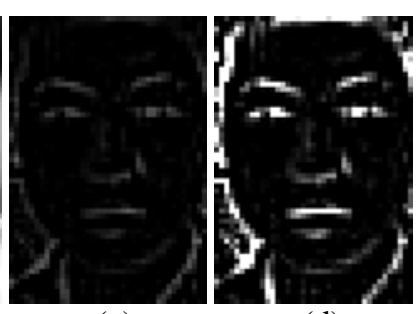

(c) (d)
Figure 3. (a) is source image (b) and (c) are S and G image.(d) is I image which is for joint analysis. 

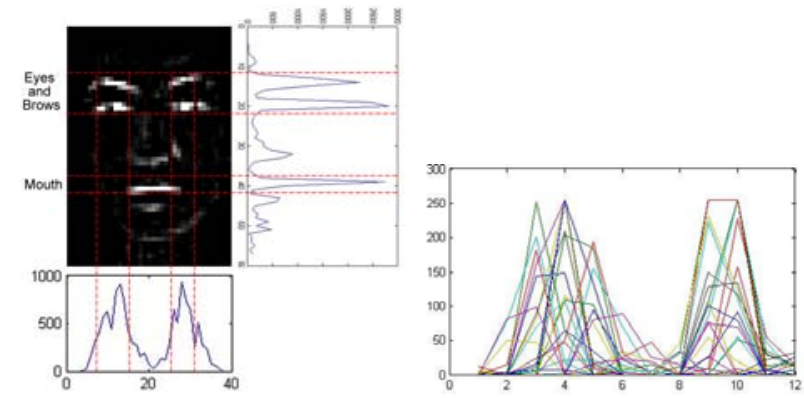

Figure 4. Projection method to locate eyes: project binary image into both $\mathrm{x}$ and y coordinate and find the peak value.

\section{Iris Positioning}

This step concerns on eyeball location. First of all the gray image is thresholded, after median filter and morphological closing are applied to it, we get several candidate regions. Then eye ball region is told apart from hair, eye bow and others by geometry, area and relative location. In the eyeball region, the image is thresholded and results in a binary image which Then Canny operator is applied to and results in the circular edge of the pupil. After noise removal and smoothing the edges, circular Hough transform is applied to locate the pupil.

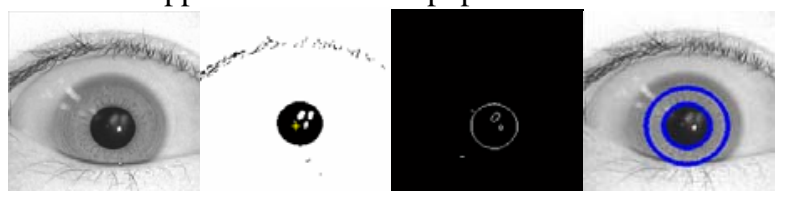

Figure 5. Steps to locate iris

\section{Iris Recognition}

The iris recognition technique applied in this work is composed of three stages: preprocessing stage, feature extraction stage and matching stage.

In the preprocessing stage, after using Canny edge detection to find edges, we applied a Hough transform to locate the iris region. Then, we remapped each point within the iris region to a pair of polar coordinates, so that the region has a fixed dimension, following which we applied a Histogram Equalization.

In the second stage, we used a Gabor filters to extraction the iris pattern and obtain a feature vector. A general 2dimension Gabor filter is shown as follow:

$$
\mathrm{G}(\mathrm{x}, \mathrm{y})=\mathrm{e}^{-\pi}\left[\left(\mathrm{x}^{-\mathrm{x}_{0}}\right)^{2} / \alpha^{2}+\left(\mathrm{y}-\mathrm{y}_{0}\right)^{2} / \beta^{2}\right] \mathrm{e}^{-2 \pi\left[\mathrm{u}_{0}\left(\mathrm{x}-\mathrm{X}_{0}\right)+\mathrm{v}_{0}\left(\mathrm{y}-\mathrm{y}_{0}\right)\right]}
$$

In the matching stage, we compared the feature vector extracted from the iris with the ones stored in the database by calculating their Vector Similarity using:

$$
d\left(x_{k}, x_{j}\right)=\frac{x_{k} \bullet x_{j}}{\left\|x_{k}\right\| *\left\|x_{j}\right\|}
$$

where $x k$ is the vector of the tested iris, $x j$ is one of the iris templates, and $d$ is the similarity of the two vectors. If the two vectors are derived from the same iris, the $d$ will be close to 1 , otherwise to 0 .

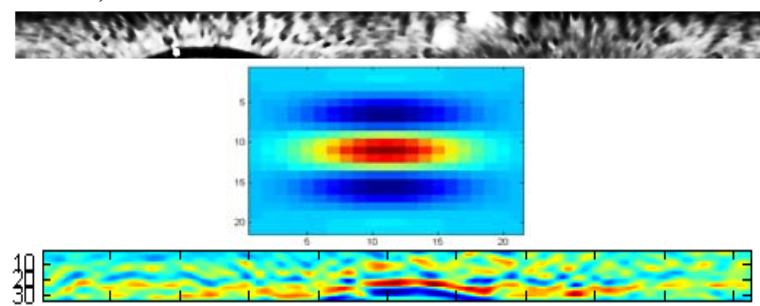

Figure 6. Iris image and the image filtered by Gabor

Ultimately, the filtered image is projected into a vector of length 512. Euler distance from vectors in the database is calculated one by one, before the matching result is given.

\section{EXPERIMENT}

We tested the Eye Targeting module in different conditions, and the results are presented in Table 1.

TABLE I. SUCCESS RATIO OF FACE DETECTION AND EYE POSITIONING UNDER DIFFERENT CONDITIONS SUCH AS DIFFERENT ILLUMINATION.

\begin{tabular}{ccc}
\hline $\begin{array}{c}\text { Testing } \\
\text { Condition }\end{array}$ & $\begin{array}{c}\text { Success Ratio of } \\
\text { Finding Face \% }\end{array}$ & $\begin{array}{c}\text { Success Ratio of } \\
\text { Locating Eyes\% }\end{array}$ \\
\hline Soft light & 100.0 & 98.5 \\
Against light & 92.5 & 78.1 \\
Large-angle & 97.3 & 65.3 \\
rotated face & 92.5 & 87.5 \\
Multi persons & 100.0 & 98.5 \\
Soft light & & \\
\hline
\end{tabular}

36 volunteers registered into our database. The testing results shown in Table 2 are based on this.

TABLE II. RATIO OF FAILURE CAUSED BY IMAGE INVALIDATION OR INTRA-CLASS SIMILARITY . ALSO DISTANCE BETWEEN USERS AND THE SYSTEM TERMINAL IS CHANGED

\begin{tabular}{ccccccc}
\hline $\begin{array}{c}\text { Cause of } \\
\text { failure/Distance }\end{array}$ & $0.5 \mathrm{~m}$ & $1 \mathrm{~m}$ & $1.5 \mathrm{~m}$ & $2 \mathrm{~m}$ & $2.5 \mathrm{~m}$ & $3 \mathrm{~m}$ \\
\hline Image Invalidation & 0.1 & 0 & 1.2 & 4.1 & 8.8 & 13.4 \\
Intra-class Similarity & 3.0 & 2.3 & 2.5 & 3.1 & 6.1 & 10.2 \\
Total & 3.1 & 2.3 & 3.7 & 7.2 & 14.9 & 23.6 \\
\hline
\end{tabular}

\section{ACKNOWLEDGEMENT}

This work was supported by National Natural Science Foundation of China Grant No. 61201425.

\section{REFERENCES}

[1] Daugman J, Probing the uniqueness and randomness of IrisCodes: Results from 200 billion iris pair comparisons, IEEE, 2006 vol. 94, no. 11, pp 1927-1935

[2] Daugman J and Downing C, Epigenetic randomness, complexity, and singularity of human iris patterns, Royal Society, 2001, B, 268, Biological Sciences, 2001, pp 1737 1740 , 
[3] Richard P. Wildes, Iris Recognition: An Emerging Biometric Technology, Proceedings of the IEEE,Vol 85, Issue 9, Sept. 1997, pp:1348 - 1363

[4] R. P. Wildes J. C. Asmuth G. L. Green S. C. Hsu R. J. Kolczynski J. R. Matey S. E. McBride, A System for Automated Iris Recognition,IEEE,pp.121-128

[5] Eric Sung, Towards non-cooperative iris recognition systems, IEEE, Volume 2, 2-5 Dec. 2002 ,pp:990-995

[6] R.L.Hsu. Face detection and modeling for recognition[D], Ph.D. CSE.MSU.2002

[7] Rafael C. Gonzalez and Richard E. Woods, Digital Image Processing Using MATLAB[M], publ. Pearson Education,Inc.,ISBN:0130085197,Chapter 9,pp:337-356
[8] R. P. Wildes, A System for Automated Iris Recognition,IEEE,5-7 Dec. 1994, pp:121 - 128

[9] Shimaa M. Elsherief, Biometric Personal Identification Based on Iris Recognition,Computer Engneering and Systems,IEEE,5-7 Nov. 2006, pp:208 - 213

[10] Proenca, H. Alexandre, L.A., Iris segmentation methodology for non-cooperative recognition,IEEE, Volume 153, Issue 2, 6 April 2006, pp:199-205

[11] Covavisaruch, N., Prateepamornkul, P., Personal Identification System using Hand Geometry and Iris Pattern Fusion, Electro/information Technology, 2006 IEEE International Conference on,7-10 May 2006, pp:597 - 602 